\title{
A Frame-Free Formulation of Elasticity
}

\author{
by Walter Noll, August 2005* \\ Professor Emeritus, \\ Carnegie Mellon University \\ e-mail: wn0g@andrew.cmu.edu
}

\section{Introduction}

The term "principle of material frame-indifference" was introduced in 1965 by C. Truesdell and me in our contribution [NLFT] to the Encyclopedia of Physics. Earlier, I had used the term "principle of objectivity", and some people use this term to this day. I meant "objectivity" to express independence of the "observer", but Truesdell disliked the term as being too easily misinterpreted. In fact, I now believe that the principle has nothing to do with "observer", who is defined in the dictionary to be a person. What matters is the use of frames of reference as a means to make precise the concept of location, as explained in Sect.4 of $[\mathrm{N} 2]$ of $[\mathrm{FC}] . * *$

Here is a full statement of the principle of material frame-indifference, as it applies to any physical system:

The constitutive laws governing the internal interactions between the parts of the system should not depend on whatever external frame of reference is used to describe them.

The principle applies only to external frames of reference, not to frames that are constructed from the system itself. It is important to note that the principle applies only to internal interactions, not to actions of the environment on the system and its parts, because usually the frame of reference employed is actively connected with the environment. For example, if one considers the motion of a fluid in a container, one usually uses the frame of reference determined by the container, which certainly affects the fluid. Inertia should always be considered as an action of the environment on the given system and its parts, and hence its description does depend on the frame of reference used.

Remark: It has been pointed out by Ingo Müller *** that some of the equations of the thermo-mechanics of rarified gases derived from statistical mechanics do not satisfy the principle of frame-indifference. These formulas involve inertial

* This paper is based, in part, on lectures that I gave on June 29, 2005 at the meeting in Reggio-Calabria in honor of the 65th birthday of Gianpietro Del Piero and on July 6, 2005, at the University of Messina.

** I regret that I ever used the term "principle of objectivity" and thereby misled a lot of people. I came to a complete understanding of the principle of frame-indifference only in the past 15 years or so.

$* * *$ in a lecture at the meeting in Reggio-Calabria mentioned above. 
effects not captured by the standard formula, in which all the inertial forces are propertional to the mass density. This failure of frame-indifference is not surprising because inertial actions are external actions and not internal interactions. Since the mean free path between collisions of molecules in rarified gases can be very large, it is doubtful that interactions such as forces and heattransfer can be described as pure surface interactions as in standard continuum thermo-mechanics. (For a rigorous explanation, see [N6].) Therefore, formulas that formally look like internal constitutive equations may actually have a non-standand conceptual interpretation. I

First, in Sect.1 below, I will describe the conventional formulation of the constitutive equation of elasticity and the effect of the principle of frame-indifference on it. I believe that this description is more transparent than the one given in $[\mathrm{NLFT}]$ and the standard textbooks because it makes explicit use of different frame-spaces.

As I pointed out at the end of Sect.4 in [N2], it should be possible to make the principle of material frame-indifference vacuously satisfied by using an intrinsic mathematical frame-work that does not use an external frame-space at all when describing the internal interactions of a physical system. In Sects. 2 and 3 below I will do just that for the classical theory of elasticity by specializing the treatment given in Sects.7, 9, and 10 of [N7]. In Sect.4 I will do the same for hyperelasticity, i.e., elasticity based on a strain-energy function. (See Sect.82 of [NLFT].) In Sect.5 I will comment on possible restrictions on the intrinsic response functions defined in Sects.2 and 4.

As I pointed out in [NO] of [FC], I believe that [NLFT] is now in many respects obsolete and should be updated. In the third paper [N3] of [FC] I present some guidelines for such an update. The present paper could serve as a blueprint for updating Chapter D of [NLFT] and also for the beginning chapters of future textbooks on elasticity.

In the remainder of this paper, we assume that a continuous body-system $\mathcal{B}$ as described in Sect.3 of [N3] is given. The tangent space of $\mathcal{B}$ at a material point $X \in \mathcal{B}$ is denoted by $\mathcal{T}_{X}$ and is called the body element of $\mathcal{B}$ at $X$. We say that the body system consists of a simple material if the constitutive laws for every material point $X$ involve only the body element $\mathcal{T}_{X}$. A precise way of formulating such laws is to put them in the form of a mathematical structure involving $\mathcal{T}_{X}$. Most of the time we single out a particular material point $X$ and simply write $\mathcal{T}$ instead of $\mathcal{T}_{X}$. We emphasize that $\mathcal{T}$ is a linear space but not an inner-product space.

We use the mathematical infrastructure, notation, and terminology of [FDS]. In particular, we use "lineon" as a contraction of "linear transformation from a linear space to itself". *)

*) In [NLFT] the term "tensor" is used instead of "lineon". I pointed out in $[\mathrm{N} 8]$ that "tensor" has a much more general meaning and lineon is just a special case. 


\section{Conventional Elasticity}

The conventional formulation of the theory of elasticity involves a frame space $\mathcal{F}$ (usually just called "space"), a reference placement $\kappa$ of $\mathcal{B}$ whose framespace is $\mathcal{F}$, and, for each material point $X$, a response function $\mathfrak{g}: \mathcal{L} \longrightarrow \operatorname{Sym} \mathcal{V}$, where $\mathcal{L}$ is a suitable open subset of the linear group Lis $\mathcal{V}$ of all automorphisms of the translation space $\mathcal{V}$ of $\mathcal{F}$, i.e. all invertible lineons on $\mathcal{V}$. It is assumed that $\mathbf{1}_{\mathcal{V}}$, the identity mapping of $\mathcal{V}$ and the unity of the group Lis $\mathcal{V}$, belong to $\mathcal{L}$. The value

$$
\mathbf{T}:=\mathfrak{g}(\mathbf{F})
$$

is the Cauchy-stress at the present when $\mathbf{F}:=\nabla_{\kappa(X)} \chi_{t}$ is the gradient at $\kappa(X)$ of the transplacement $\chi_{t}$ from the reference placement $\kappa$ to the present placement

$$
\mu_{t}:=\chi_{t} \circ \kappa .
$$

The dependence of $\mathfrak{g}$ on the material point $X$ and the dependence of $\mathbf{F}$ and $\mathbf{T}$ on time need not be made explicit. (See Sect.43 of [NLFT].)

Now consider two frames of reference with corresponding frame-spaces $\mathcal{F}$ and $\mathcal{F}^{\prime}$ and denote their translation spaces by $\mathcal{V}$ and $\mathcal{V}^{\prime}$, respectively. If $x$ is the location of a material point at a given time $t$ in the frame-space $\mathcal{F}$, then the location of the same material point at the same time $t$ in the frame-space $\mathcal{F}^{\prime}$ will be given by $x^{\prime}=\alpha_{t}(x)$ where $\alpha_{t}: \mathcal{F} \longrightarrow \mathcal{F}^{\prime}$ is an isometry and hence a Euclidean isomorphism (see Sect.45 of [FDS]). The mapping $t \mapsto \alpha_{t}$ describes the motion of the frame $\mathcal{F}$ relative to the frame $\mathcal{F}^{\prime}$. The present placement relative to $\mathcal{F}^{\prime}$ corresponding to the placement $\mu_{t}$ relative to $\mathcal{F}$ is given by

$$
\mu_{t}^{\prime}=\alpha_{t} \circ \mu_{t}
$$

The gradient $\mathbf{A}_{t}:=\nabla \alpha_{t} \in \operatorname{Lis}\left(\mathcal{V}, \mathcal{V}^{\prime}\right)$ (see Sect.33 of [FDS]) is an inner-product preserving linear isomorphism from $\mathcal{V}$ onto $\mathcal{V}^{\prime}$. A vector $\mathbf{u}$ and a lineon $\mathbf{L}$ relative to the frame-space $\mathcal{F}$ at time $t$ will appear as

$$
\mathbf{u}^{\prime}=\mathbf{A}_{t} \mathbf{u} \text { and } \mathbf{L}^{\prime}=\mathbf{A}_{t} \mathbf{L} \mathbf{A}_{t}^{-1},
$$

respectively, relative to the frame-space $\mathcal{F}^{\prime}$. In particular, the present Cauchystress $\mathbf{T}^{\prime}$ relative to $\mathcal{F}^{\prime}$ is related to the present Cauchy-stress $\mathbf{T}$ relative to $\mathcal{F}$ by

$$
\mathbf{T}^{\prime}=\mathbf{A}_{t} \mathbf{T} \mathbf{A}_{t}^{-1}, \text { where } \mathbf{A}_{t}=\nabla \alpha_{t} .
$$

To say that descriptions in two frame spaces $\mathcal{F}$ and $\mathcal{F}^{\prime}$ describe the same physical process means that these descriptions must be isomorphic, i.e., the description in the frame $\mathcal{F}$ can be transported to the description in the frame $\mathcal{F}^{\prime}$ by a fixed Euclidean isomorphism $\beta: \mathcal{F} \longrightarrow \mathcal{F}^{\prime}$. Its gradient $\mathbf{B}:=\nabla \beta \in \operatorname{Lis}\left(\mathcal{V}, \mathcal{V}^{\prime}\right)$ is an inner-product preserving linear isomorphism from $\mathcal{V}$ to $\mathcal{V}^{\prime}$ 
Now let the same elastic response at a material point desribed by (1.1) in the frame $\mathcal{F}$ be described by the response function $\mathfrak{g}^{\prime}: \mathcal{T}^{\prime} \longrightarrow$ Sym $\mathcal{V}^{\prime}$ in the frame $\mathcal{F}^{\prime}$. The requirement that this response function is obtained from the original one by the Euclidean isomorphism $\beta$ has the following consequences:

(a) The reference placement $\kappa^{\prime}$ used for $\mathfrak{g}^{\prime}$ must be given by

$$
\kappa^{\prime}=\beta \circ \kappa \text {. }
$$

(b) The domain $\mathcal{L}^{\prime}$ of response function $\mathfrak{g}^{\prime}$ must be related to the domain of the response function $\mathfrak{g}$ by

$$
\mathcal{L}^{\prime}=\left\{\mathbf{B L B}^{-1} \mid \mathbf{L} \in \mathcal{L}\right\} \subset \text { Lis } \mathcal{V}^{\prime}
$$

and we must have

$$
\mathfrak{g}^{\prime}\left(\mathbf{B L B}^{-1}\right)=\mathbf{B g}(\mathbf{L}) \mathbf{B}^{-1} \quad \text { for all } \mathbf{L} \in \mathcal{L} .
$$

Now, in the frame $\mathcal{F}^{\prime}$, the transplacement $\chi_{t}^{\prime}$ from the reference placement $\kappa^{\prime}$ to the present placement $\mu_{t}^{\prime}$ is characterized by

$$
\mu_{t}^{\prime}=\chi_{t}^{\prime} \circ \kappa^{\prime} \text {. }
$$

Using (1.3), (1.2), and (1.6), it follows from (1.9) that $\alpha_{t} \circ \mu_{t}=\alpha_{t} \circ \chi_{t} \circ \kappa=$ $\chi_{t}^{\prime} \circ \beta \circ \kappa$ and hence that $\alpha_{t} \circ \chi_{t}=\chi_{t}^{\prime} \circ \beta$. Taking the gradient at $\kappa(X)$, we obtain

$$
\mathbf{A}_{t} \mathbf{F}=\mathbf{F}^{\prime} \mathbf{B},
$$

where $\mathbf{F}^{\prime}$ is the gradient at $\kappa^{\prime}(X)=\beta(\kappa(X))$ of the transplacement $\chi_{t}^{\prime}$.

For the frame $\mathcal{F}^{\prime}$, the stress relation (1.1) becomes

$$
\mathbf{T}^{\prime}:=\mathfrak{g}^{\prime}\left(\mathbf{F}^{\prime}\right) \text {. }
$$

Using (1.5),(1.1) and (1.10), it follows from (1.11) that

$$
\mathbf{A}_{t} \mathfrak{g}(\mathbf{F}) \mathbf{A}_{\mathfrak{t}}^{-1}=\mathfrak{g}^{\prime}\left(\mathbf{A}_{\mathfrak{t}} \mathbf{F B}^{-1}\right) .
$$

Using (1.8) with $\mathbf{L}:=\mathbf{B}^{-1} \mathbf{A}_{t} \mathbf{F}$, we obtain

$$
\mathfrak{g}^{\prime}\left(\mathbf{A}_{\mathfrak{t}} \mathbf{F B}^{-1}\right)=\mathbf{B} \mathfrak{g}\left(\mathbf{B}^{-1} \mathbf{A}_{\mathfrak{t}} \mathbf{F}\right) \mathbf{B}^{-1}
$$

and hence, by (1.12),

$$
\mathbf{Q} \mathfrak{g}(\mathbf{F}) \mathbf{Q}^{\top}=\mathfrak{g}(\mathbf{Q F}) \text {, where } \mathbf{Q}:=\mathbf{B}^{-1} \mathbf{A}_{\mathfrak{t}} .
$$

We note that $\mathbf{Q}$ is an inner-product automorphism of $\mathcal{V}$, in other words, a member of the orthogonal group Orth $\mathcal{V}$, so that $\mathbf{Q}^{-1}=\mathbf{Q}^{\top}$. The dependence of $\mathbf{F}$ and $\mathbf{Q}$ on time need not be made explicit here. Now, (1.13) must hold for arbitrary motions and hence for all $\mathbf{F} \in \mathcal{L}$. Also, the frame $\mathcal{F}^{\prime}$ can be chosen in an arbitrary manner and hence (1.13) must also hold for all $\mathbf{Q} \in$ Orth $\mathcal{V}$. Thus, the relation (1.13) coincides with (43.2) in [NLFT] and is the basis for all the reduced constitutive equations stated in Sect.43 of [NLFT]. 


\section{Frame-free Elasticity}

To describe the structure of an elastic element, we need a little bit of linear algebra that is not presented in most textbooks. (However, a detailed treatment is given in Chapter 2 of [FDS].)

Let a finite-dimensional linear space $\mathcal{T}$ be given. The dual $\mathcal{T}^{*}:=\operatorname{Lin}(\mathcal{T}, \mathbb{R})$ of $\mathcal{T}$ consists of all linear forms on $\mathcal{T}$. The dual $\mathcal{T}^{* *}$ of the dual $\mathcal{T}^{*}$ can be identified with the original space $\mathcal{T}$ as follows: When an element $\mathbf{t} \in \mathcal{T}$ is regarded as an element of $\mathcal{T}^{* *}$, its action on $\mathcal{T}^{*}$ is given by

$$
\mathbf{t} \lambda:=\lambda \mathbf{t} \text { for all } \lambda \in \mathcal{T}^{*} .
$$

We express this identification by $\mathcal{T}^{* *} \cong \mathcal{T}$.

Let $\mathcal{V}$ also be finite-dimensional linear space and let $\mathbf{L} \in \operatorname{Lin}(\mathcal{T}, \mathcal{V})$ be a linear mapping from $\mathcal{T}$ to $\mathcal{V}$. Then the transpose $\mathbf{L}^{\top} \in \operatorname{Lin}\left(\mathcal{V}^{*}, \mathcal{T}^{*}\right)$ is defined by

$$
\left(\mathbf{L}^{\top} \boldsymbol{\lambda}\right) \mathbf{t}:=\boldsymbol{\lambda}(\mathbf{L} \mathbf{t}) \text { for all } \mathbf{t} \in \mathcal{T}, \boldsymbol{\lambda} \in \mathcal{V}^{*} .
$$

Let $\mathbf{G} \in \operatorname{Lin}\left(\mathcal{T}, \mathcal{T}^{*}\right)$ be given. Then, since $\mathcal{T}^{* *} \cong \mathcal{T}$, we can identify $\operatorname{Lin}\left(\mathcal{T}^{* *}, \mathcal{T}^{*}\right) \cong \operatorname{Lin}\left(\mathcal{T}, \mathcal{T}^{*}\right)$, so that $\mathbf{G}$ and its transpose $\mathbf{G}^{\top}$ belong to the same space. We say that $\mathbf{G}$ is symmetric if $\mathbf{G}^{\top}=\mathbf{G}$. The set

$$
\operatorname{Sym}\left(\mathcal{T}, \mathcal{T}^{*}\right):=\left\{\mathbf{G} \in \operatorname{Lin}\left(\mathcal{T}, \mathcal{T}^{*}\right) \mid \mathbf{G}^{\top}=\mathbf{G}\right\}
$$

is a subspace of $\operatorname{Lin}\left(\mathcal{T}, \mathcal{T}^{*}\right)$.

We say that $\mathbf{G} \in \operatorname{Sym}\left(\mathcal{T}, \mathcal{T}^{*}\right)$ is positive if

$$
(\mathbf{G t}) \mathbf{t} \geq 0 \text { for all } \mathbf{t} \in \mathcal{T}
$$

and we say that it is strictly positive if, in addition, (Gt)t is zero only when $\mathbf{t}$ is zero. The set of all positive elements of $\operatorname{Sym}\left(\mathcal{T}, \mathcal{T}^{*}\right)$ is denoted by $\operatorname{Pos}\left(\mathcal{T}, \mathcal{T}^{*}\right)$ and the set of all strictly positive elements by $\operatorname{Pos}^{+}\left(\mathcal{T}, \mathcal{T}^{*}\right)$. Both $\operatorname{Pos}\left(\mathcal{T}, \mathcal{T}^{*}\right)$ and $\operatorname{Pos}^{+}\left(\mathcal{T}, \mathcal{T}^{*}\right)$ are linear cones, i.e. they are stable under addition and scalar multiplication with strictly positive real numbers*. All the elements of $\operatorname{Pos}^{+}\left(\mathcal{T}, \mathcal{T}^{*}\right)$ are invertible and hence linear isomorphisms, i.e., we have

$$
\operatorname{Pos}^{+}\left(\mathcal{T}, \mathcal{T}^{*}\right) \subset \operatorname{Lis}\left(\mathcal{T}, \mathcal{T}^{*}\right)
$$

An inner-product space is a finite-dimensional linear space $\mathcal{V}$ with an additional structural element given by singling out a particular member ip $\in \operatorname{Pos}\left(\mathcal{T}, \mathcal{T}^{*}\right)$. Since ip $\in \operatorname{Lis}\left(\mathcal{V}, \mathcal{V}^{*}\right)$, it then can be and is used to identify $\mathcal{V}^{*} \cong \mathcal{V}$, which justifies the standard notation $\mathbf{v} \cdot \mathbf{w}:=\mathbf{i p}(\mathbf{v}) \mathbf{w}$ for all $\mathbf{v}, \mathbf{w} \in \mathcal{V}$.

As explained in Sects. 3 and 4 of [N3], a configuration of the given bodysystem $\mathcal{B}$ is a metric $d: \mathcal{B} \times \mathcal{B} \longrightarrow \mathbb{P}$ that is isometric to a fit region** in a

* A detailed analysis of such linear cones is given in [NS].

** The definition and a detailed analysis of the concept of a fit region is given in $[\mathrm{NV}]$. 
Euclidean space. Such a configuration induces an element $\mathbf{G} \in \operatorname{Pos}^{+}\left(\mathcal{T}, \mathcal{T}^{*}\right)$ for the body element $\mathcal{T}$. We call $\mathbf{G}$ the configuration of the element induced by the configuration $d$ of the whole system.

The structure of an elastic material element is described by the following ingredients:

1) A body element $\mathcal{T}$,

2) A convex open subset $\mathcal{G}$ of $\operatorname{Pos}^{+}\left(\mathcal{T}, \mathcal{T}^{*}\right)$, whose members are called the configurations of the element.

3) A mapping $\mathfrak{h}: \mathcal{G} \longrightarrow \operatorname{Sym}\left(\mathcal{T}^{*}, \mathcal{T}\right)$, called the

intrinsic stress-response function of the element. Its value

$$
\mathbf{S}=\mathfrak{h}(\mathbf{G})
$$

is the intrinsic stress determined by the configuration $\mathbf{G}$.

A deformation of the elastic element is simply a change of configurations, and a deformation process is a one-paramter family of configurations as described in Sect.4 of [N3].

As I pointed out in Sect.6 of [N3], a frame of reference is usually employed to describe the external actions of the environment on the body-system. Thus, we now assume, as in Sect.1, that a frame space $\mathcal{F}$ with translation space $\mathcal{V}$ and a reference placement $\kappa$ of $\mathcal{B}$ in $\mathcal{F}$ are given. We denote the present placement by $\mu_{t}$ and the transplacement from the reference placement $\kappa$ to the present placement by $\chi_{t}$, so that (1.2) holds. Taking the gradient of (1.2) at the given material point $X \in \mathcal{B}$ we obtain

$$
\mathbf{M}=\mathbf{F K}, \text { where } \mathbf{K}:=\nabla_{X} \kappa, \mathbf{M}:=\nabla_{X} \mu_{t} \text {, and } \mathbf{F}:=\nabla_{\kappa(X)} \chi_{t} .
$$

We call $\mathbf{K} \in \operatorname{Lis}(\mathcal{T}, \mathcal{V})$ the reference placement of the body element $\mathcal{T}$, $\mathbf{M} \in \operatorname{Lis}(\mathcal{T}, \mathcal{V})$ the present placement of this body element, and $\mathbf{F} \in \operatorname{Lis}(\mathcal{V})$ the transplacement of this body element from $\mathbf{K}$ to $\mathbf{M}$. The dependence of $\mathbf{M}$ and $\mathbf{F}$ on time need not be made explicit.

As explained in Sect.4 of [N3], the configuration

$$
\mathbf{G}_{R}:=\mathbf{K}^{\top} \mathbf{K} \in \operatorname{Pos}^{+}\left(\mathcal{T}, \mathcal{T}^{*}\right)
$$

of the element $\mathcal{T}$ is induced by the reference placement $\mathbf{K}$ and will be called the reference configuration. The present configuration

$$
\mathbf{G}:=\mathbf{M}^{\top} \mathbf{M}=\mathbf{K}^{\top} \mathbf{F}^{\top} \mathbf{F K} \in \operatorname{Pos}^{+}\left(\mathcal{T}, \mathcal{T}^{*}\right)
$$

of the element $\mathcal{T}$ is induced by the present placement $\mathbf{M}$. The requirement that $\mathbf{G}_{R}$ and $\mathbf{G}$ belong to the prescribed subset $\mathcal{G}$ of $\operatorname{Pos}^{+}\left(\mathcal{T}, \mathcal{T}^{*}\right)$ is equivalent to the conditions

$$
\mathbf{F} \in \mathcal{L}:=\left\{\mathbf{F} \in \operatorname{Lis} \mathcal{V} \mid \mathbf{K}^{\top} \mathbf{F}^{\top} \mathbf{F} \mathbf{K} \in \mathcal{G}\right\} \text { and } \mathbf{1}_{\mathcal{V}} \in \mathcal{L}
$$


Now, by (6.4) of [N3], the present Cauchy-stress $\mathbf{T}$ is related to the present intrinsic stress $\mathbf{S}$ by

$$
\mathbf{S}=\mathbf{M}^{-1} \mathbf{T} \mathbf{M}^{\top^{-1}}
$$

Therefore, since $\mathbf{S}=\mathfrak{h}(\mathbf{G})$ is the intrinsic stress determined by the present configuration $\mathbf{G}$, it follows from (2.11), (2.9) and (2.7) that the Cauchy stress determined by $\mathbf{F}$ is given by

$$
\mathbf{T}=\mathfrak{g}(\mathbf{F}):=\mathbf{F K h}\left(\mathbf{K}^{\top} \mathbf{F}^{\top} \mathbf{F K}\right) \mathbf{K}^{\top} \mathbf{F}^{\top} .
$$

The domain $\mathcal{L}$ of the response function $\mathfrak{g}$ defined by (2.12) is given by (2.10). Given $\mathbf{F} \in \mathcal{L}$ it easily follows from (2.12) that $\mathfrak{g}$ does indeed satisfy the condition (1.13) for all $\mathbf{Q} \in \operatorname{Orth} \mathcal{V}$.

We note that the intrinsic response function $\mathfrak{h}$ is related to the response function $\mathfrak{t}$ for the second Piola-Kirchhoff tensor (see $(43.10)_{2}$ in [NLFT])as follows:

$$
\tilde{\mathbf{T}}=\mathfrak{t}(\mathbf{C})=\sqrt{(\operatorname{det} \mathbf{C})} \mathbf{K} \mathfrak{h}\left(\mathbf{K}^{\top} \mathbf{C K}\right) \mathbf{K}^{\top} \text { when } \mathbf{C}:=\mathbf{F}^{\top} \mathbf{F} .
$$

We emphasize again that the body-element $\mathcal{T}$ is just a three-dimensional linear space and not an inner product space. Let a configuration $\mathbf{G}$ of $\mathcal{T}$ be given. It is a member of the linear cone $\operatorname{Pos}^{+}\left(\mathcal{T}, \mathcal{T}^{*}\right)$. Since an inner product is just a member of this cone that has been singled out, we can deal with $\mathbf{G}$ as if it were an inner product and apply the the concepts, notations, and theory of innerproduct spaces, but they must be used only relative to the given configuration. For example, a basis $\mathbf{b}:=\left(\mathbf{b}_{1}, \mathbf{b}_{2}, \mathbf{b}_{3}\right)$ of $\mathcal{T}$ is $\mathbf{G}$-orthonormal if

$$
\left(\mathbf{G b}_{i}\right) \mathbf{b}_{j}=\delta_{i, j}:=\left\{\begin{array}{ll}
0 & \text { if } i \neq j \\
1 & \text { if } i=j
\end{array} \quad \text { for all } \quad i, j \in\{1,2,3\}\right.
$$

A basis that is $\mathbf{G}$-orthonormal necessarily fails to be $\mathbf{G}^{\prime}$ - orthonormal when $\mathbf{G}^{\prime}$ is a configuration different from $\mathbf{G}$.

The orthogonal group of the configuration $\mathbf{G}$ is defined by

$$
\operatorname{Orth}(\mathbf{G}):=\left\{\mathbf{A} \in \operatorname{Lis} \mathcal{T} \mid \mathbf{A}^{\top} \mathbf{G} \mathbf{A}=\mathbf{G}\right\}
$$

$\operatorname{Orth}(\mathbf{G})$ is not only a subgroup of the linear group Lis $\mathcal{T}$, but even of the unimodular group

$$
\operatorname{Unim} \mathcal{T}:=\{\mathbf{A} \in \operatorname{Lis} \mathcal{T}|| \operatorname{det} \mathbf{A} \mid=1\} .
$$

Unim $\mathcal{T}$ includes infinitely many orthogonal groups as subgroups, namely one for each configuration $\mathbf{G}$.

Let $\mathbf{S}$ be the intrinsic stress determined by the configuration $\mathbf{G}$ as in (2.6). The spectral theory as treated, for example, in Chapter 8 of [FDS] can be applied 
to $\mathbf{S}$ relative to $\mathbf{G}$ with the following result: There is a $\mathbf{G}$-orthonormal basis $\mathbf{b}:=\left(\mathbf{b}_{1}, \mathbf{b}_{2}, \mathbf{b}_{3}\right)$ of $\mathcal{T}$ and a list $\left(\sigma_{1}, \sigma_{2}, \sigma_{3}\right)$ of real numbers such that

$$
(\mathbf{S G}) \mathbf{b}_{i}=\sigma_{i} \mathbf{b}_{i} \quad \text { for all } i \in\{1.2 .3\} .
$$

The terms in $\left(\sigma_{1}, \sigma_{2}, \sigma_{3}\right)$ are called the principal stresses of $\mathbf{S}$ relative to $\mathbf{G}$.

If we introduce a frame-space $\mathcal{F}$ as before and consider the Cauchy-stress $\mathbf{T}$ related to $\mathbf{S}$ by (2.11), it follows from (2.17), using (2.9), that

$$
\mathbf{T v}_{i}=\sigma_{i} \mathbf{v}_{i}, \text { where } \mathbf{v}_{i}:=\mathbf{M} \mathbf{b}_{i} \text { for all } i \in\{1.2 .3\} .
$$

Hence the principal stresses characterized by (2.17) coincide with the ones in conventional elasticity. (See Sect.48 of [NLFT].)

\section{Material Isomorphisms, Material Symmetry}

Isomorphisms between elastic material elements are defined in accordance with the general notion of isomorphism for arbitrary mathematical structures. To be specific, let $\left(\mathcal{T}_{1}, \mathcal{G}_{1}, \mathfrak{h}_{1}\right)$ and $\left(\mathcal{T}_{2}, \mathcal{G}_{2}, \mathfrak{h}_{2}\right)$ be the ingredients that define two elastic material elements as described by 1$), 2$ ), and 3 ) in the previous section. A linear isomorphism $\mathbf{A} \in \operatorname{Lis}\left(\mathcal{T}_{1}, \mathcal{T}_{2}\right)$ is a material isomorphism if

$$
\mathcal{G}_{1}=\left\{\mathbf{A}^{\top} \mathbf{G} \mathbf{A} \mid \mathbf{G} \in \mathcal{G}_{2}\right\}
$$

and

$$
\mathbf{A} \mathfrak{h}_{1}\left(\mathbf{A}^{\top} \mathbf{G A}\right) \mathbf{A}^{\top}=\mathfrak{h}_{2}(\mathbf{G}) \text { for all } \mathbf{G} \in \mathcal{G}_{2} .
$$

We say that an elastic body system $\mathcal{B}$ is materially uniform if, for any two material points $X, Y \in \mathcal{B}$, the corresponding elastic elements $\left(\mathcal{T}_{X}, \mathcal{G}_{X}, \mathfrak{h}_{X}\right)$ and $\left(\mathcal{T}_{Y}, \mathcal{G}_{Y}, \mathfrak{h}_{Y}\right)$ are materially isomorphic. We say that the body system $\mathcal{B}$ is homogeneous if there is a placement $\kappa$ of $\mathcal{B}$ in a frame-space $\mathcal{F}$ with translation space $\mathcal{V}$ such that, for any two material points $X, Y \in \mathcal{B}$,

$$
\mathbf{A}_{X Y}:=\left(\nabla_{Y} \kappa\right)^{-1} \nabla_{X} \kappa \in \operatorname{Lis}\left(\mathcal{T}_{X}, \mathcal{T}_{Y}\right)
$$

is a material isomorphism.

A body system can be materially uniform without being homogeneous, and the deviation from homogeneity is related to what are often called "continuous distributions of dislocations". (See also the Remark at the end of Sect.9 in [N7].)

Now let an elastic material element $(\mathcal{T}, \mathcal{G}, \mathfrak{h})$ be given as described in the previous section. An automorphism of this element, i.e., an isomorphism from the element to itself, is called a symmetry of the element. We assume that all symmetries are unimodular because a change of volume cannot reasonably leave material properties unchanged. They form the symmetry group

$$
\mathfrak{G}:=\left\{\mathbf{A} \in \operatorname{Unim} \mathcal{T} \mid \mathbf{A} \mathfrak{h}\left(\mathbf{A}^{\top} \mathbf{G A}\right) \mathbf{A}^{\top}=\mathfrak{h}(\mathbf{G}) \text { for all } \mathbf{G} \in \mathcal{G}\right\}
$$


Of course, it must be assumed that, for each $\mathbf{A} \in \mathfrak{G}$, we must have $\mathbf{A}^{\top} \mathbf{G} \mathbf{A} \in \mathcal{G}$ whenever $\mathbf{G} \in \mathcal{G}$.

The symmetry group of a configuration $\mathbf{G} \in \mathcal{G}$ is defined by

$$
\mathfrak{G}_{\mathbf{G}}:=\mathfrak{G} \cap \operatorname{Orth}(\mathbf{G})
$$

It is easily seen that

$$
\mathbf{A} \mathfrak{h}(\mathbf{G}) \mathbf{G}=\mathfrak{h}(\mathbf{G}) \mathbf{G A} \quad \text { for all } \quad \mathbf{A} \in \mathfrak{G}_{\mathbf{G}} .
$$

We say that a given configuration $\mathbf{G} \in \mathcal{G}$ is isotropic if $\mathfrak{G}_{\mathbf{G}}:=\operatorname{Orth}(\mathbf{G})$, i.e., if $\operatorname{Orth}(\mathbf{G}) \subset \mathfrak{G}$. We denote the set of all isotropic configurations by

$$
\operatorname{Itr} \mathcal{G}:=\{\mathbf{G} \in \mathcal{G} \mid \operatorname{Orth}(\mathbf{G}) \subset \mathfrak{G}\} .
$$

If $\mathbf{G} \in \mathcal{G}$ is isotropic, it follows from (3.5) that $\mathfrak{h}(\mathbf{G}) \mathbf{G}$ commutes with all $\mathbf{A} \in \operatorname{Orth}(\mathbf{G})$ and hence is proportional to the identity $\mathbf{1}_{\mathcal{T}}$ of $\mathcal{T}$. Therefore, there is a function $\hat{\pi}: \operatorname{Itr} \mathcal{G} \longrightarrow \mathbf{R}$ such that

$$
\mathfrak{h}(\mathbf{G}) \mathbf{G}=-\hat{\pi}(\mathbf{G}) \mathbf{1}_{\mathcal{T}} \quad \text { for all } \mathbf{G} \in \operatorname{Itr} \mathcal{G} .
$$

It easily follows from (3.3), (3.6) and (3.7) that

$$
\hat{\pi}\left(\mathbf{A}^{\top} \mathbf{G A}\right)=\hat{\pi}(\mathbf{G}) \quad \text { for all } \mathbf{G} \in \operatorname{Itr} \mathcal{G}, \mathbf{A} \in \mathfrak{G} .
$$

We need the following

Proposition. Let $\mathbf{G}, \mathbf{G}^{\prime} \in \operatorname{Pos}^{+}\left(\mathcal{T}, \mathcal{T}^{*}\right)$ be given. Then

$$
\left(\mathbf{G}^{\prime}=\mathbf{A}^{\top} \mathbf{G A} \text { for some } \mathbf{A} \in \operatorname{Unim} \mathcal{T}\right) \Longleftrightarrow \operatorname{det}\left(\mathbf{G}^{-1} \mathbf{G}^{\prime}\right)=1 .
$$

The proof of this proposition is essentially the same as that of Prop.19.1 in $[\mathrm{N} 7]$.

Using this Proposition and (3.8), we see that

$$
\left(\operatorname{det}\left(\mathbf{G}^{-1} \mathbf{G}^{\prime}\right)=1 \Longrightarrow \hat{\pi}\left(\mathbf{G}^{\prime}\right)=\hat{\pi}(\mathbf{G})\right) \text { for all } \mathbf{G}, \mathbf{G}^{\prime} \in \operatorname{Itr} \mathcal{G} \text {. }
$$

We now choose a reference configuration $\mathbf{G}_{R} \in \mathcal{G}$ and define the function $\hat{\rho}: \mathcal{G} \longrightarrow \mathbb{P}^{\times}$by

$$
\hat{\rho}(\mathbf{G})=\sqrt{\operatorname{det}\left(\mathbf{G}^{-1} \mathbf{G}_{R}\right)} \quad \text { for all } \mathbf{G} \in \mathcal{G} .
$$

If the units for mass and volume are chosen such that the mass density (mass per unit volume) in the reference configuration is 1 , then the value $\hat{\rho}(\mathbf{G})$ is the mass-density determined by the configuration G. Here, mass can be taken as a proxy for volume in the reference-configuration, and hence need not be 
connected with inertial or gravitational mass, which should not enter internal constitutive laws. It is easily seen from (3.11) that

$$
\operatorname{det}\left(\mathbf{G}^{-1} \mathbf{G}^{\prime}\right)=1 \Longleftrightarrow \hat{\rho}\left(\mathbf{G}^{\prime}\right)=\hat{\rho}(\mathbf{G}) \quad \text { for all } \mathbf{G}, \mathbf{G}^{\prime} \in \mathcal{G} .
$$

Therefore, by (3.10), the value $\hat{\pi}(\mathbf{G})$ depends only on $\hat{\rho}(\mathbf{G})$. Hence there is a function

$$
\bar{\pi}: P \longrightarrow \mathbf{R}, \text { where } P:=\hat{\rho}_{>}(\operatorname{Itr} \mathcal{G}),
$$

such that $\hat{\pi}(\mathbf{G})=\bar{\pi}(\hat{\rho}(\mathbf{G}))$ for all $\mathbf{G} \in \operatorname{Itr} \mathcal{G}$. Hence (3.7) yields

$$
\mathfrak{h}(\mathbf{G})=-\bar{\pi}(\hat{\rho}(\mathbf{G})) \mathbf{G}^{-1} \quad \text { for all } \mathbf{G} \in \operatorname{Itr} \mathcal{G} .
$$

The function $\bar{\pi}$ is called the pressure function for the set $\operatorname{Itr} \mathcal{G}$ of all isotropic configurations of the elastic material element.

We now assume, as at the end of Sect.2, that a frame space $\mathcal{F}$ with translation space $\mathcal{V}$ and a reference placement $\kappa$ of $\mathcal{B}$ in $\mathcal{F}$ are given and we use again the notation (2.7). It easily follows from (2.8) and (2.9) that

$$
\operatorname{det}\left(\left(\mathbf{G}_{R}\right)^{-1} \mathbf{G}\right)=\operatorname{det}\left(\mathbf{F} \mathbf{F}^{\top}\right)=|\operatorname{det} \mathbf{F}|^{2}
$$

and hence, by (3.11), $\hat{\rho}(\mathbf{G})=|\operatorname{det} \mathbf{F}|^{-1}$. Therefore, the value $\bar{\rho}(\mathbf{F})$ of the function $\bar{\rho}: \mathcal{L} \longrightarrow \mathbb{P}^{\times}$defined by

$$
\bar{\rho}(\mathbf{F}):=|\operatorname{det} \mathbf{F}|^{-1} \quad \text { for all } \mathbf{F} \in \mathcal{L}
$$

gives the mass-density of the body element when its configuration is determined by $\mathbf{F}$ according to (2.9). Using (2.12), (3.14), and (2.9), we easily conclude that

$$
\mathbf{T}=\mathfrak{g}(\mathbf{F})=-\bar{\pi}\left(\bar{\rho}(\mathbf{F}) \mathbf{1}_{\mathcal{V}}\right.
$$

when the configuration determined by $\mathbf{F}$ is isotropic. This formula (3.16) is consistent with conventional formulas such as $(50.2)_{1}$ in $[\mathrm{NLFT}]$.

We say that the element is fluid if its symmetry group is the full unimodular group, i.e., if

$$
\mathfrak{G}=\operatorname{Unim} \mathcal{T} .
$$

The element is fluid if and only if all of its configurations are isotropic, i.e., we have $\operatorname{Itr} \mathcal{G}=\mathcal{G}$. Hence, for a fluid element, the relation (3.14) is valid for all configurations $\mathbf{G} \in \mathcal{G}$ and (3.16) is valid for all $\mathbf{F} \in \mathcal{L}$. In this case, the pressure function $\bar{\pi}$ alone is enough to describe the elastic response.

We say that the element is solid if

$$
\mathfrak{G} \subset \operatorname{Orth}(\mathbf{G}) \text { for some } \mathbf{G} \in \mathcal{G} \text {. }
$$

If that is the case then the configurations $\mathbf{G}$ for which (3.18) holds are said to be undistorted. 
We say that the element is isotropic if it has isotropic configurations, i.e.,

$$
\operatorname{Orth}(\mathbf{G}) \subset \mathfrak{G} \text { for some } \mathbf{G} \in \mathcal{G}
$$

Assume now on that the element is an isotropic solid element, which means, by (3.18) and (3.19), that its undistorted configurations $\mathbf{G}$ are characterized by the property that

$$
\operatorname{Orth}(\mathbf{G})=\mathfrak{G}
$$

In fact, for isotropic solids, the set of undistorted configurations coincides with the set $\operatorname{Itr} \mathcal{G}$ of isotropic configurations. Moreover, if $\mathbf{G}_{R}$ is an undistorted reference configuration, we have

$$
\operatorname{Orth}\left(\mathbf{G}_{R}\right)=\mathfrak{G}=\operatorname{Orth}(\mathbf{G}) \quad \text { for all } \mathbf{G} \in \operatorname{Itr} \mathcal{G}
$$

It easily follows from (3.21) that all undistorted configurations are proportional to $\mathbf{G}_{R}$, i.e., that

$$
\operatorname{Itr} \mathcal{G} \subset\left\{c \mathbf{G}_{R} \mid c \in \mathbb{P}^{\times}\right\}
$$

\section{Frame-free Hyperelasticity}

The structure of a hyperelastic material element is obtained from that of an elastic material element defined in Sect.2 by adding two more ingredients and a basic axiom. The ingredients are:

4) A reference configuration $\mathbf{G}_{R} \in \mathcal{G}$.

5) A function $\hat{\sigma}: \mathcal{G} \longrightarrow \mathbb{P}$, of class $\mathrm{C}^{1}$, called the intrinsic energy-

response function.

Its value

$$
\sigma=\hat{\sigma}(\mathbf{G})
$$

is the strain-energy per unit mass determined by the configuration $\mathbf{G}$.

As in the previous section, mass can be taken as a proxy for volume in the reference configuration.

To formulate the basic axiom of hyperelasticity, we need the following result from linear algebra, which is not in most textbooks:

Every member $\overline{\mathbf{M}}$ of $\left(\operatorname{Sym}\left(\mathcal{T}, \mathcal{T}^{*}\right)\right)^{*}:=\operatorname{Lin}\left(\operatorname{Sym}\left(\mathcal{T}, \mathcal{T}^{*}\right), \mathbb{R}\right)$ has exactly one representation by a member $\mathbf{M}$ of $\operatorname{Sym}\left(\mathcal{T}^{*}, \mathcal{T}\right)$ such that

$$
\overline{\mathbf{M}}(\mathbf{L})=\operatorname{tr}(\mathbf{M L}) \quad \text { for all } \mathbf{L} \in \operatorname{Sym}\left(\mathcal{T}, \mathcal{T}^{*}\right)
$$

(This result is a consequence of the Representation Theorem for Linear Forms on a Space of Linear Mappigs in Sect 26 of FDS.)

We use $(4.2)$ to identify $\left(\operatorname{Sym}\left(\mathcal{T}, \mathcal{T}^{*}\right)\right)^{*} \cong \operatorname{Sym}\left(\mathcal{T}^{*}, \mathcal{T}\right)$ by omitting, on the right side of (4.2), the bar atop $\mathbf{M}$. 
To formulate the basic axiom, we also make use of the mass-density function $\hat{\rho}$ defined by (3.11).

Basic Axiom: The intrinsic stress-response function is related to the intrinsic energy response function by

$$
\mathfrak{h}(\mathbf{G})=2 \widehat{\rho}(\mathbf{G}) \nabla_{\mathbf{G}} \hat{\sigma} \quad \text { for all } \mathbf{G} \in \mathcal{G} .
$$

Note that (4.3) makes sense only because of the identification mediated by (4.2) when the bar over $\mathbf{M}$ is omitted because the right side belongs to $\left(\operatorname{Sym}\left(\mathcal{T}, \mathcal{T}^{*}\right)\right)^{*}$ while the left side belongs to $\operatorname{Sym}\left(\mathcal{T}^{*}, \mathcal{T}\right)$.

We now assume that a frame-vector-space $\mathcal{V}$ is given as in previous sections. We choose a reference placement $\mathbf{K} \in \operatorname{Lis}(\mathcal{T}, \mathcal{V})$ of the element in such a way that

$$
\mathbf{G}_{R}:=\mathbf{K}^{\top} \mathbf{K},
$$

the configuration induced by $\mathbf{K}$, is the reference configuration given as ingredient in 4). Every other placement of the element in the space $\mathcal{V}$ then has the form $\mathbf{F K} \in \operatorname{Lis}(\mathcal{T}, \mathcal{V})$ where $\mathbf{F} \in \operatorname{Lis} \mathcal{V}$ is a transplacement of the element. The configuration $\mathbf{G}$ induced by the placement $\mathbf{F K}$ is

$$
\mathbf{G}=(\mathbf{F K})^{\top}(\mathbf{F K})=\mathbf{K}^{\top} \mathbf{C K}, \text { where } \mathbf{C}:=\mathbf{F}^{\top} \mathbf{F} .
$$

Recall that $\mathbf{C}$ is called the right Cauchy-Green tensor in [NLFT].

We now put

$$
\overline{\mathcal{G}}:=\left\{\mathbf{C} \in \operatorname{Pos}^{+} \mathcal{V} \mid \mathbf{K}^{\top} \mathbf{C K} \in \mathcal{G}\right\}
$$

and define $\bar{\sigma}: \overline{\mathcal{G}} \longrightarrow \mathbb{P}^{\times}$by

$$
\bar{\sigma}(\mathbf{C}):=\hat{\sigma}\left(\mathbf{K}^{\top} \mathbf{C K}\right) \quad \text { for all } \quad \mathbf{C} \in \overline{\mathcal{G}} .
$$

It easily follows from (4.7) that

$$
\nabla_{\mathbf{C}} \bar{\sigma}=\mathbf{K}\left(\nabla_{\mathbf{K}^{\top} \mathbf{C K}} \hat{\sigma}\right) \mathbf{K}^{\top} \quad \text { for all } \quad \mathbf{C} \in \overline{\mathcal{G}} .
$$

In analogy to (4.7) define $\bar{\rho}: \overline{\mathcal{G}} \longrightarrow \mathbb{P}^{\times}$by

$$
\bar{\rho}(\mathbf{C}):=\hat{\rho}\left(\mathbf{K}^{\top} \mathbf{C K}\right) \quad \text { for all } \quad \mathbf{C} \in \overline{\mathcal{G}} .
$$

Now put

$$
\mathcal{L}:=\left\{\mathbf{F} \in \operatorname{Lis} \mathcal{V} \mid \mathbf{F}^{\top} \mathbf{F} \in \overline{\mathcal{G}}\right\} .
$$

In view of $(2.12)$ we have

$\mathfrak{g}(\mathbf{F}):=(\mathbf{F K}) \mathfrak{h}\left(\mathbf{K}^{\top} \mathbf{C K}\right)(\mathbf{F K})^{\top}$, where $\mathbf{C}:=\mathbf{F}^{\top} \mathbf{F}, \quad$ for all $\mathbf{F} \in \mathcal{L}$ 
Therefore, by (4.3), (4.7), (4.8), and (4.9), we conclude that

$\mathfrak{g}(\mathbf{F}):=\rho(\mathbf{C}) \mathbf{F}\left(\nabla_{\mathbf{C}} \sigma\right) \mathbf{F}^{\top}$, where $\mathbf{C}:=\mathbf{F}^{\top} \mathbf{F}, \quad$ for all $\mathbf{F} \in \mathcal{L}$.

This relation is identical to (84.12) in [NLFT] and hence shows that the basic axiom is consistent with the classical frame-dependent theory of hyperelasticity.

Remark: The relation (4.12) is a reduced version of the stress relation (82.9) in [NLFT]. In the context of thermodynamics, the latter can be derived from the second law of thermodynamics by a procedure described in $[\mathrm{CN}]$ (see (5.4) in $[\mathrm{CN}])$. A frame-free version of this procedure should yield the relation (4.3) of the Basic Axiom directly.

The symmetry group of the strain-energy is defined by

$$
\mathfrak{G}_{\sigma}:=\left\{\mathbf{A} \in \operatorname{Unim} \mathcal{T} \mid \hat{\sigma}\left(\mathbf{A}^{\top} \mathbf{G A}\right)=\hat{\sigma}(\mathbf{G}) \text { for all } \mathbf{G} \in \mathcal{G}\right\} .
$$

Theorem. We have $\mathfrak{G}_{\sigma} \subset \mathfrak{G}$, i.e., every strain-energy symmetry is also a symmetry of the elastic element. If the element is solid and if the reference confiuration is undistorted we actually have $\mathfrak{G}_{\sigma}=\mathfrak{G}$.

Proof: Let $\mathbf{A} \in \mathfrak{G}_{\sigma}$ be given so that

$$
\hat{\sigma}\left(\mathbf{A}^{\top} \mathbf{G} \mathbf{A}\right)=\hat{\sigma}(\mathbf{G}) \text { for all } \mathbf{G} \in \mathcal{G} .
$$

Taking the gradient with respect to $\mathbf{G}$ and using the identification (4.2), we find

$$
\begin{gathered}
\operatorname{tr}\left(\left(\nabla_{\mathbf{G}} \hat{\sigma}\right) \mathbf{L}\right)=\operatorname{tr}\left(\left(\nabla_{\mathbf{A}^{\top} \mathbf{G} \mathbf{A}} \hat{\sigma}\right) \mathbf{A}^{\top} \mathbf{L} \mathbf{A}\right)= \\
\operatorname{tr}\left(\mathbf{A}\left(\nabla_{\mathbf{A}^{\top} \mathbf{G} \mathbf{A}} \hat{\sigma}\right) \mathbf{A}^{\top} \mathbf{L}\right) \quad \text { for all } \mathbf{L} \in \operatorname{Sym}\left(\mathcal{T}, \mathcal{T}^{*}\right),
\end{gathered}
$$

and hence

$$
\mathbf{A}\left(\nabla_{\mathbf{A}^{\top} \mathbf{G} \hat{\mathbf{A}}^{\sigma}}\right) \mathbf{A}^{\top}=\nabla_{\mathbf{G}} \sigma .
$$

Noting, in view of (3.11), that

$$
\hat{\rho}\left(\mathbf{A}^{\top} \mathbf{G} \mathbf{A}\right)=\hat{\rho}(\mathbf{G}) \text { for all } \mathbf{G} \in \mathcal{G},
$$

it follows from (4.16) and the basic axiom (4.3) that $\mathbf{A} \mathfrak{h}\left(\mathbf{A}^{\top} \mathbf{G A}\right) \mathbf{A}^{\top}=\mathfrak{h}(\mathbf{G})$ for all $\mathbf{G} \in \mathcal{G}$ and hence, in view of (3.3), that $\mathbf{A} \in \mathfrak{G}$. Since $\mathbf{A} \in \mathfrak{G}_{\sigma}$ was arbitrary, the inclusion $\mathfrak{G}_{\sigma} \subset \mathfrak{G}$ is proved.

Actually, the proof above shows that $\mathbf{A} \in \mathfrak{G}$ if and only if the gradient of (4.14) with respect to $\mathbf{G}$ is zero. That is the case if and only if the difference $\hat{\sigma}\left(\mathbf{A}^{\top} \mathbf{G A}\right)-\hat{\sigma}(\mathbf{G})$ does not depend on $\mathbf{G}$. This means that we have $\mathbf{A} \in \mathfrak{G}$ if and only if

$$
\hat{\sigma}\left(\mathbf{A}^{\top} \mathbf{G A}\right)-\hat{\sigma}(\mathbf{G})=\hat{\sigma}\left(\mathbf{A}^{\top} \mathbf{G}_{R} \mathbf{A}\right)-\hat{\sigma}\left(\mathbf{G}_{R}\right) \text { for all } \mathbf{G} \in \mathcal{G} .
$$


Now, if the element is solid and the reference configuration undistorted, it follows from (3.16) with $\mathbf{G}:=\mathbf{G}_{R}$ that $\mathbf{A} \in \operatorname{Orth}\left(\mathbf{G}_{R}\right)$. Therefore, we have $\mathbf{A}^{\top} \mathbf{G}_{R} \mathbf{A}=$ $\mathbf{G}_{R}$ and the right side of (4.18) is zero. Since $\mathbf{A} \in \mathfrak{G}$ was arbitrary we conclude from (4.18) and (4.14) that, in this case, $\mathfrak{G}_{\sigma}=\mathfrak{G}$.

The Theorem just proved is the frame-free version of a result presented in Sect.58 of [NLFT].

\section{Restrictions upon the Response Functions.}

The most common restrictions on the intrinsic response functions concern smoothness. If the energy response function $\hat{\sigma}$ is of class $\mathrm{C}^{1}$ then the stress response function $\mathfrak{h}$ is continuous; if $\hat{\sigma}$ is of class $C^{2}$, then $\mathfrak{h}$ is of class $C^{1}$. The latter assumption is sufficient for most purposes.

The linear cone $\operatorname{Pos}^{+}\left(\mathcal{T}, \mathcal{T}^{*}\right)$ and the space $\operatorname{Sym}\left(\mathcal{T}, \mathcal{T}^{*}\right)$ have natural (partial) orders, denoted by $\prec$ and defined by

$$
\mathbf{G}_{1} \prec \mathbf{G}_{2} \Longleftrightarrow \mathbf{G}_{2}-\mathbf{G}_{1} \in \operatorname{Pos}^{+}\left(\mathcal{T}, \mathcal{T}^{*}\right) \text { for all } \mathbf{G}_{1}, \mathbf{G}_{2} \in \operatorname{Pos}^{+}\left(\mathcal{T}, \mathcal{T}^{*}\right)
$$

and

$$
\mathbf{S}_{1} \prec \mathbf{S}_{2} \Longleftrightarrow \mathbf{S}_{2}-\mathbf{S}_{1} \in \operatorname{Pos}^{+}\left(\mathcal{T}^{*}, \mathcal{T}\right) \text { for all } \mathbf{S}_{1}, \mathbf{S}_{2} \in \operatorname{Sym}\left(\mathcal{T}^{*}, \mathcal{T}\right)
$$

(These definitions are special cases of (2.8) in [NS].)

Note: Given $\mathbf{G} \in \operatorname{Pos}^{+}\left(\mathcal{T}, \mathcal{T}^{*}\right)$ and $c \in \mathbb{P}^{\times}$we have $\mathbf{G} \prec c \mathbf{G} \Longleftrightarrow c>1$.

We postulate the following

Axiom. The stress-response function $\mathfrak{h}$ is strictly isotone in the sense that

$$
\mathbf{G}_{1} \prec \mathbf{G}_{2} \Longrightarrow \mathfrak{h}\left(\mathbf{G}_{1}\right) \prec \mathfrak{h}\left(\mathbf{G}_{2}\right) \text { for all } \mathbf{G}_{1}, \mathbf{G}_{2} \in \mathcal{G} \text {. }
$$

Intuitively, the axiom states that in order to expand the elastic element in all directions, one must increase the stresses in all directions.

Proposition. For isotropic materials, the pressure function $\bar{\pi}$ characterized by (3.13) and (3.14) is stricty antitone in the sense that

$$
\rho_{1}<\rho_{2} \Longrightarrow \bar{\pi}\left(\rho_{1}\right)>\bar{\pi}\left(\rho_{2}\right) \text { for all } \rho_{1}, \rho_{2} \in P .
$$

Proof: Let $\rho_{1} \in P$ be given. By (3.13), we may choose $\mathbf{G}_{1} \in \operatorname{Itr} \mathcal{G}$ such that $\rho_{1}=\hat{\rho}\left(\mathbf{G}_{1}\right)$. Let $c \in \mathbb{P}^{\times}$be given and put $\mathbf{G}_{2}:=c \mathbf{G}_{1} \in \mathcal{G}$. Since $\operatorname{Orth}\left(\mathbf{G}_{2}\right)=$ $\operatorname{Orth}\left(\mathbf{G}_{1}\right) \subset \mathfrak{G}$ we also have $\mathbf{G}_{2} \in \operatorname{Itr} \mathcal{G}$. An easy calculation using (3.11) shows that

$$
\hat{\rho}\left(\mathbf{G}_{2}\right)=c^{-\frac{3}{2}} \hat{\rho}\left(\mathbf{G}_{1}\right)=c^{-\frac{3}{2}} \rho_{1} .
$$

Now let $\rho_{2} \in P$ be given. By (5.5) we then have

$$
\hat{\rho}\left(\mathbf{G}_{2}\right)=\rho_{2} \quad \Longleftrightarrow \quad c:=\left(\frac{\rho_{1}}{\rho_{2}}\right)^{\frac{2}{3}} .
$$


We assume now that (5.6) holds and use (3.14) with the result

$$
\mathfrak{h}\left(\mathbf{G}_{2}\right)=-\bar{\pi}\left(\rho_{2}\right) \mathbf{G}_{2}^{-1}=-\bar{\pi}\left(\rho_{2}\right)\left(c \mathbf{G}_{1}\right)^{-1}=\frac{\bar{\pi}\left(\rho_{2}\right)}{\bar{\pi}\left(\rho_{1}\right)} c^{-1} \mathfrak{h}\left(\mathbf{G}_{1}\right) .
$$

Using (5.6) we see that

$$
\mathbf{G}_{1} \prec \mathbf{G}_{2} \Longleftrightarrow c>1 \Longleftrightarrow \rho_{1}>\rho_{2}
$$

and using (5.7) we see that

$$
\mathfrak{h}\left(\mathbf{G}_{1}\right) \prec \mathfrak{h}\left(\mathbf{G}_{2}\right) \Longleftrightarrow \frac{\bar{\pi}\left(\rho_{2}\right)}{\bar{\pi}\left(\rho_{1}\right)}>c .
$$

Combining (5.8) and (5.9), we infer from the strict isotonicity of $\mathfrak{h}$ that $\rho_{1}>\rho_{2} \Longrightarrow \bar{\pi}\left(\rho_{2}\right)>\bar{\pi}\left(\rho_{1}\right)$.

The antitonicity of $\bar{\pi}$ implies that there is at most one $\rho \in P$ such that $\bar{\pi}(\rho)=0$. In view of (3.20) we conclude that, for isotropic solids, there is at most one undistorted configuration $\mathbf{G}_{N}$ such that $\mathfrak{h}\left(\mathbf{G}_{N}\right)=\mathbf{0}$. If such $\mathbf{G}_{N}$ exists, it is called a natural configuration.

A response restriction that one might explore is the assumption that the energy-response function $\hat{\sigma}$ is convex. It is not hard to prove that this is the case if and only if the stress-response function $\mathfrak{h}$ satisfies

$$
\operatorname{tr}\left(\left(\rho_{1} \mathfrak{h}\left(\mathbf{G}_{2}\right)-\rho_{2} \mathfrak{h}\left(\mathbf{G}_{1}\right)\right)\left(\mathbf{G}_{2}-\mathbf{G}_{1}\right)\right) \geq 0 \quad \text { for all } \mathbf{G}_{1}, \mathbf{G}_{2} \in \mathcal{G}
$$

when $\rho_{1}:=\hat{\rho}\left(\mathbf{G}_{1}\right), \rho_{2}:=\hat{\rho}\left(\mathbf{G}_{2}\right)$.

A large number of possible restrictions on the response functions have been considered in the conventional literature on elasticity. The following names appear in Sects. 51, 52, and 86 of [NLFT]: P-C inequality, T-E inequalities, IFS condition, E-T inequalities, $O-F$ inequalities, $B$ - $E$ inequalities, E-inequalities, $G C N$ condition, and $C-N$ condition. In the later literature, conditions such as rank-one convexity, quasi-convexity, and poly-convexity have been extensively explored.

All these restrictions should be frame-indifferent in order to be relevant. I am not sure whether this has been proved in all cases. If they are frameindifferent, it must be possible to give them a frame-free formulation. I hope that someone will start the task of doing so in the future.

\section{Phase Transitions.}

The domain $\mathcal{G}$ of the intrinsic response function $\mathfrak{h}$ in Sect.2 (or of the intrinsic strain energy function $\hat{\sigma}$ in Sect.4) must be restricted in such a way that the configurations in $\mathcal{G}$ are compatible with only a single phase of the material element in question. If the material element undergoes a phase transition, the 
configurations of the new phase belong to a subset $\mathcal{G}^{\prime}$ of $\operatorname{Pos}^{+}\left(\mathcal{T}, \mathcal{T}^{*}\right)$ that is disjoint from $\mathcal{G}$. The intrinsic response function $\mathfrak{h}^{\prime}$ for the new phase need not have the same material symmetry as $\mathfrak{h}$. For example, the material can be fluid in one phase and solid in the other. If a configuration belongs to a point on the boundary of both $\mathcal{G}$ and $\mathcal{G}^{\prime}$, one can expect that the element can describe two coexistent phases.

Phase transitions are related to questions of stability, and these are related to the restrictions mentioned in the previous section. I hope that someone will develop a frame-free conceptual analysis of such phase transitions. This analysis cannot be complete unless the entire matter is treated in the context of thermomechanics.

Acknowledgement: I am grateful to Roger Fosdick for helpful suggestions and proofreading.

\section{References}

[FDS] Noll, W. :Finite-Dimensional Spaces: Algebra, Geometry, and Analysis, Vol.I, 393 pages, posted on the website math.cmu.edu/ wnog/noll . ( Originally published by Martinus Nijhoff Publishers in 1987.)

[NLFT] Truesdell, C. \& Noll, W.: The Non-Linear Field Theories of Mechan$i c s$, third Edition, 602 pages. Springer-Verlag, 2004. (The first edition appeared in 1965.)

[FC] Noll, W. : Five Contributions to Natural Pilosophy, 73 pages 2004, posted on the website math.cmu.edu/ wnOg/noll .

[N0] Noll, W. Introduction to [FC]

[N1] Noll, W. On the Illusion of Physical Space, first paper in [FC]

[N2] Noll, W.On the Principle of Material Frame-Indifference, second paper in $[\mathrm{FC}]$.

[N3] Noll, W.: Updating the The Non-Linear Field Theories of Mechanics, third paper in $[\mathrm{FC}]$.

[N6] Noll, W.: The Theory of Surface Interactions, to be published (2005)

[N7] Noll, W.: A new mathematical theory of simple materials, Archive for Rational Mechanics and Analysis 52, 1-50 (1972).

[N8] Noll, W.: Isocategories and Tensor Functors, 19 pages (1992). Posted on the website math.cmu.edu/ wnOg/noll .

[NS] Noll, W \& Schäffer, J. J.: Orders, gauge, and distance in faceless linear cones; with examples relevant to continuum mechanics and relativity, Archive for Rational Mechanics and Analysis 66, 345-377 (1977).

[NV] Noll, W. \& Virga, E.: Fit Regions and Functions of Bounded Variation, Archive for Rational Mechanics and Analysis 102, 1-21 (1988).

[CN] Coleman, B.D. \& Noll, W.: The thermodynamics of elastic materials with heat conduction and viscosity, Archive for Rational Mechanics and Analysis, 13, 167-178 (1963). 